\title{
Nel regno del possibile. Incontri tra fotografia e poesia nell'opera di Mario Giacomelli
}

\author{
Giovanni NASSI \\ Monza \\ ORCID: 00oo-0002-4522-9090
}

\begin{abstract}
Lo studio prende in considerazione la pratica di Mario Giacomelli di comporre serie fotografiche in relazione a testi poetici. Vengono indagati in particolare gli intrecci semantici generati dall'incontro tra gli scatti del maestro marchigiano e i versi di Mario Luzi.
\end{abstract}

Keywords: Mario Giacomelli, Mario Luzi, La notte lava la mente, ipertesto visuale, transito.

\begin{abstract}
Parole e immagini sono complementari e si cercano eternamente le une con le altre.

Johann Wolfgang von Goethe
\end{abstract}

\section{Tentativi intermediali ${ }^{\mathrm{I}}$}

L'opera di Mario Giacomelli (Senigallia I925-200o) all'interno della storia della fotografia occupa una posizione di rilievo a livello internazionale ${ }^{2}$, in virtù di uno stile estremamente personale e riconoscibile che gli ha assicurato l'interesse da parte della critica e un susseguirsi di mostre e pubblicazioni che a vent'anni dalla scomparsa del fotografo non accenna a diminuire.

Nonostante ciò, gli studi dedicati all'opera del maestro marchigiano si sono poco soffermati attorno a una delle sue caratteristiche più peculiari, ovvero alla consuetudine di comporre sequenze di immagini in relazione a testi poetici. L'attenzione dei critici ha preferito focalizzarsi in modo approfondito sulla straordinaria capacità di trasfigurazione della realtà ritratta in fase di ripresa e in camera oscura, mentre il legame con le poesie da cui

\footnotetext{
I L'autore desidera ringraziare Katiuscia Biondi, curatrice dell'Archivio Mario Giacomelli ( Rita Giacomelli, Simone Giacomelli, direttore dell'Archivio Mario Giacomelli @ Simone Giacomelli e Simona Guerra per l'accesso alle fonti e per il prezioso confronto che ha sostenuto il presente lavoro.

2 Fortuna che ha inizio a partire dalla scelta di John Szarkowski di acquisire i suoi scatti per il dipartimento di fotografia del MoMA di New York e di ospitare la fotografia dal titolo Il bambino di Scanno all'interno della fondamentale mostra The photographer's eye nel 1964.
} 
spesso Giacomelli ha tratto i titoli delle sue serie fotografiche sembra essere stato ritenuto trascurabile al fine della visione delle stampe, come esplicitamente sostenuto da Paolo Morello che parla di «un uso spesso molto superficiale [...] della poesia» (2010: 395) o dal fotografo Piergiorgio Branzi, per cui le stesse immagini «erano già espressione poetica[,] non abbisognavano di sottolineature che risultavano stranianti, pretestuose, una ricerca di legittimazione, provincialismo insomma» (Guerra 2007: II3).

L'importanza del legame intertestuale per Giacomelli al fine della fruizione delle immagini è testimoniata dalla costante presenza delle liriche di riferimento nelle sue pubblicazioni e nelle mostre, oltre a essere stata ribadita in diverse occasioni dallo stesso fotografo:

Leggere la poesia prima di aver visto le mie fotografie rende certamente le cose più facili, perché se io parlo di qualcosa che tu non conosci è naturale che tu non capisca: alcune immagini potrebbero piacerti, però potresti anche interpretarle in un altro modo rispetto a come erano state suggerite. Io ero legato alla poesia! Quindi questa permette di capirle; senza potresti dire: «Ah, quanto è bella questa fotografia!», e magari le altre no. Ogni immagine è il supporto per un'altra. È come fare le scale: uno scalino alla volta! Quindi è naturale che sia più semplice poterle guardare accompagnate alla poesia (Guerra 2008: I65-166).

Intento di questo lavoro è dare credito all'operazione dell'autore raccogliendo l'invito, attraverso un approccio interdisciplinare, ad avventurarsi nel terreno liminare dell'incontro tra versi e fotografie per valutare quale esperienza di lettura ne scaturisce.

Considerando la vasta produzione giacomelliana si nota che nelle diciassette serie a cui, a partire dagli anni Sessanta, il fotografo ha attribuito il titolo di una poesia ${ }^{3}$, il rapporto con i diversi testi si sia declinato mediante approcci molteplici, le cui tappe principali sono state recentemente rilevate da Marco Andreani (2019). Di fondamentale importanza per l'elaborazione dei primi tentativi di incontro intermediale negli anni Sessanta è la frequentazione di Giacomelli con Luigi Crocenzi, fotografo di Fermo che si cimentava nella composizione di fotoracconti ${ }^{4}$ sul «Politecnico» di Elio Vittorini, con il quale aveva lavorato anche sull'edizione illustrata di Conversazione in Sicilia. È infatti su suggerimento di Crocenzi che Giacomelli intraprende la realizzazione delle prime serie fotografiche in accostamento a testi poetici; in Io non ho mani che mi accarezzino il volto e Verrà la morte e avrà $i$ tuoi occhi,

3 L'elenco è riportato in Appendice.

4 Crocenzi pubblicò in Italia, tra il 1946 e il I947, i primi racconti fotografici costituiti da una sequenza di immagini alternate da testi che delineavano una storia. Per un approfondimento si veda Amodeo et al. (2013). 
egli rinomina lavori già intrapresi senza determinare un ordine compositivo consolidato, mentre nei primi anni Settanta con Caroline Branson si rileva il tentativo di veri e propri fotoracconti poetici in cui le immagini seguono un preciso criterio diegetico.

A partire dalle serie dedicate ai testi del poeta friulano Francesco Permunian a metà degli anni Ottanta, la composizione dei racconti fotografici diviene più articolata; come analizzato da Katiuscia Biondi (2OII: I3-2I), immagini realizzate su un arco di tempo pluridecennale e afferenti a diversi progetti vengono ricollocate all'interno di nuovi contesti assumendo nuova vita e significato. L'approccio ai testi, inoltre, diviene tendenzialmente più libero, meno vincolato da un'intenzione di tipo illustrativo, rinvenibile invece in prove precedenti poi rifiutate dall'autore.

Per mettere alla prova, nel corso del presente studio, l'opportunità ermeneutica di una lettura intertestuale dei lavori di Giacomelli si è scelto di approfondire l'analisi di un'unica serie composta tra il I994 e il I995 che trae il titolo da una poesia di Mario Luzi, La notte lava la mente ${ }^{5}$, pubblicata nel I957 in chiusura della raccolta Onore del vero. Sin da una prima visione l'accostamento degli scatti con il testo, invece che chiarificare il senso delle immagini, pare condurre a una moltiplicazione delle domande e degli enigmi.

\section{La notte lava la mente}

La serie La notte lava la mente, nella versione in cui è stata esposta per la prima volta nel I997, consta di ventotto fotografie in bianco e nero stampate ai sali di bromuro d'argento in formato $30 \times 40 \mathrm{~cm}$ e numerate sul retro per indicare l'ordine di esposizione ${ }^{6}$. Giacomelli decise di lavorare autonomamente senza coinvolgere Luzi nella produzione della serie fotografica (i due, infatti, si incontrarono di persona solo nel 2000 a Senigallia) e in merito alle intenzioni che sottendono l'opera ha affermato che il suo «"commento" non

5 La serie è stata esposta nel 1997 presso le gallerie Photo \& Co. di Torino, Peliti di Roma, Agathe Gaillard di Parigi e Photology di Milano che curò la pubblicazione (un calendario con due foto per ogni mese) di ventiquattro delle ventotto fotografie presenti nell'esposizione (Giacomelli 1997). Successivamente è stata riproposta integralmente in occasione dell'ampia retrospettiva dal titolo Mario Giacomelli. The black is waiting for the white presso il Metropolitan Museum of Photography di Tokyo nel 20I3. Una selezione di sedici immagini è stata pubblicata con alcune variazioni dell'ordine in Giacomelli (200I: 85-89 e 2009: 242-243). Attualmente l'intera serie è conservata presso l'Archivio Mario Giacomelli @ Rita Giacomelli sul cui sito web sono visibili dodici fotografie la cui scelta viene periodicamente rinnovata: https://www. archiviomariogiacomelli.it/I994-95-la-notte-lava-la-mente/ (consultato il oI.II.2020).

6 Come segnalato da Katiuscia Biondi in un colloquio personale, sono quarantotto gli esemplari che compongono la serie, escluse le variazioni di stampa. Il fotografo era solito operare cambiamenti nel corso del tempo per quanto riguarda la scelta e l'ordine delle immagini, poiché le serie non erano sequenze da ritenersi definitive e univoche, bensì organismi soggetti a metamorfosi. 
è una trasposizione o trascrizione del testo per immagini», quanto piuttosto il tentativo "di "rubare" quasi delle sensazioni che provengono dalla poesia» (Ria I997: 44).

Per intraprendere dunque la lettura delle immagini è bene prendere le mosse dai versi della poesia:

La notte lava la mente.

Poco dopo si è qui come sai bene,

fila d'anime lungo la cornice,

chi pronto al balzo, chi quasi in catene.

Qualcuno sulla pagina del mare

traccia un segno di vita, figge un punto.

Raramente qualche gabbiano appare

(1998: 252).

A livello tematico il testo presenta un accostamento di tre momenti differenti, ognuno corrispondente a una strofa, caratterizzati da una grande efficacia iconica e, al contempo, avvolti da un'atmosfera straniante, conferita soprattutto dalla mancanza di coordinate spazio-temporali e dalla vaghezza dei referenti pronominali. Il risultato è un componimento enigmatico, la cui chiave di lettura si trova nel richiamo del terzo verso a un contesto purgatoriale. Lo stesso Luzi nel 1990 intitolò Purgatorio. La notte lava la mente una messa in scena della seconda cantica da lui curata, composta per la maggior parte da una scelta di passi danteschi affiancati da pochi interventi di mano luziana, tra cui i versi della lirica che dà il titolo all'opera.

A guidare la visione, oltre alla scelta del titolo, Giacomelli inviò degli appunti a Davide Faccioli, curatore della prima mostra tenutasi alla Galleria Photology di Milano il 23 gennaio del 1997, in occasione dell'inaugurazione dell'esposizione:

[Mario Luzi] mi ha suggerito il valore tra spazio e tempo, tra il prima e il poi, il transito, il sospeso, le oscillazioni fra due identità uguali; per questo ho messo due foto, vanno lette ed esposte quasi unite, staccate fra loro da un soffio. Questa idea di due immagini in una è stata per me un'invenzione, capire ad esempio il transito di nube in nube, tra pioggia e pioggia (M.[ascellani] 1997:37).

La scelta di esporre le fotografie secondo una soluzione binaria era già stata praticata in precedenza, ma in questo caso ne viene esplicitata la funzione semantica in rapporto alla poesia, sottolineando come elemento cardine della serie le nozioni temporali di transito e sospensione tratte dal testo. 
La struttura binaria risulta evidente per la maggior parte delle coppie di immagini che compongono la serie, attuandosi a seconda dei casi sul piano geometrico-formale, su quello tematico, causale o temporale. Le coppie costituiscono frammenti visivi attraverso cui si dipana il racconto fotografico; si creano dunque nuclei narrativi di un itinerario intessuto da richiami tematici che presentano elementi di risonanza tra loro. Come per le sue altre serie realizzate a partire dagli anni Ottanta, anche questa è il risultato dell'accostamento di scatti eterogenei sia nella scelta dei soggetti sia nelle soluzioni stilistiche adottate, che spaziano dalla ripresa di matrice realista alla composizione tendente all'astratto.

Cercando nel testo elementi riconducibili alla dinamica temporale messa in luce da Giacomelli, si nota la tensione verso l'azione nelle anime «pronte al balzo», così come l'avverbio «raramente» proietta verso l'attesa di un'apparizione; assume infine peso particolare la locuzione avverbiale "poco dopo», che detta uno scarto di tempo rispetto al primo verso, connotato invece da una dimensione atemporale e assoluta.

Prestando attenzione alla collocazione del componimento all'interno della trasposizione teatrale del Purgatorio operata da Luzi, si nota che il testo compare nella prima scena dove, guidato dall'angelo nocchiero, fa il suo ingresso il Coro di anime che domanda «esiste il tempo?» e una voce fuori campo risponde «Sì, ed esiste il travaglio», dopodiché la stessa voce recita i versi della poesia (1990: 419-420). Il legame di questa lirica con la nozione di tempo si conferma di fronte alle parole del poeta fiorentino a introduzione della sua opera teatrale:

Dovevo trovare il fermento primo intorno a cui lievitasse la massa umana nel Purgatorio. Mi parve di trovarlo nel tempo. Il Purgatorio è l'unico regno e l'unica cantica in cui il tempo vige: e vige nel suo doppio potere di nostalgica rammemorazione del passato e di tormentosa remora dell'eterno su cui il desiderio, in forma di attesa sempre più bruciante si sposta. Tempo che divide dal mondo e tempo che divide da Dio (1990: 492).

La presenza nella lirica del fattore di transito, di passaggio, emerge anche nelle varianti autografe, tra le quali si legge: «Tempo tra una scomparsa ed una attesa / che ancora troppo mi convince, ancora / per abito rimetto a un tempo vago / quello che è d'oggi. Fugge il tempo giusto» (I998: I490).

Pur non figurando nella redazione finale del testo, il fatto che la parola «tempo» ricorra per ben tre volte costituisce una traccia significativa della tensione rintracciata nei versi da Giacomelli e riproposta nella serie. Sorprende, inoltre, riscontrare la vicinanza del primo verso con il contenuto degli appunti di presentazione della mostra, tanto più che l'artista marchigiano non poteva aver letto questa variante manoscritta, la cui pubblicazione risale al I998 ed è dunque posteriore all'esposizione delle immagini. 
Nella poesia non solo è presente una componente temporale che, sebbene non esplicita, è stata colta da Giacomelli, ma dall'analisi formale del componimento si evince la presenza di un determinato sistema binario rinvenibile nella bipartizione interna in due emistichi dei versi pari, oltre che nella rima alternata delle terzine ${ }^{7}$. Appare a questo punto come la lettura di Giacomelli testimoni un ascolto approfondito del testo e risulti perfettamente in linea con il contenuto dei versi, così come il modello costruttivo binario del ciclo fotografico abbia un riscontro testuale in precisi presupposti prosodici della lirica.

La visione della serie si apre con una prima fotografia i cui lati lunghi sono delimitati da due file di rettangoli che richiamano le fattezze di una pellicola; in questo modo si inaugura una sorta di cinematografia interiore dai tratti ambigui e stranianti, dove i legami che si stabiliscono esulano da criteri spazio-temporali rigorosi e sono riconducibili invece a una dimensione onirica. La trama sospesa di rimandi e allusioni si dipana a formare un racconto suddivisibile in due parti, incorniciate da un'introduzione e una conclusione affidate a sequenze di quattro immagini (tenendo conto anche della prima stampa cui si è fatto menzione) riconducibili alla tematica della pioggia, poste a iscrivere la narrazione entro una circolarità perfetta. La prima parte della serie, compresa dalla quinta alla tredicesima immagine, è incentrata su scene colte durante il giorno e presenta soggetti quali ombre, uomini e maschere; dalla quattordicesima alla ventiquattresima si svolge, invece, una sequenza dai toni serali o notturni, in cui il mare costituisce una presenza costante.

A un primo raffronto comparativo fra le stampe e il testo poetico si riscontrano alcuni evidenti punti di tangenza, a partire dal verso di apertura. L'incipit marcatamente metaforico evoca infatti un' immagine di lavacro, sottolineata foneticamente dall'uso reiterato della consonante liquida, che reca con sé nel contesto purgatoriale l'idea di un'abluzione capace di purificare la mente dai pensieri e dalle preoccupazioni della giornata appena conclusa. Questa metafora, che descrive di per sé qualcosa di invisibile, un'esperienza dell'anima, viene da Giacomelli interpretata visivamente mediante la sequenza composta dalle prime quattro immagini che introducono la serie; si tratta, infatti, di scatti in cui sono narrati figurativamente i diversi momenti di un temporale: l'ammassarsi delle nubi incorniciato dall'effetto pellicola, una casa sferzata dalla tempesta, la caduta di una pioggia luminosa e

7 Il secondo presenta un'importante cesura per un accento ribattuto tra la sesta e la settima sede che si traduce in una pausa di lettura, mentre il quarto vede entrambi gli emistichi introdotti dal pronome «chi» inframmezzati da una virgola ed è sempre la virgola a sottolineare nel sesto verso la divisione di «due entità uguali», per usare parole di Giacomelli, poiché connotati da una struttura grammaticale analoga. 
l'asfalto bagnato su cui sono disposti disordinatamente alcuni elementi tra cui delle lettere dell'alfabeto.

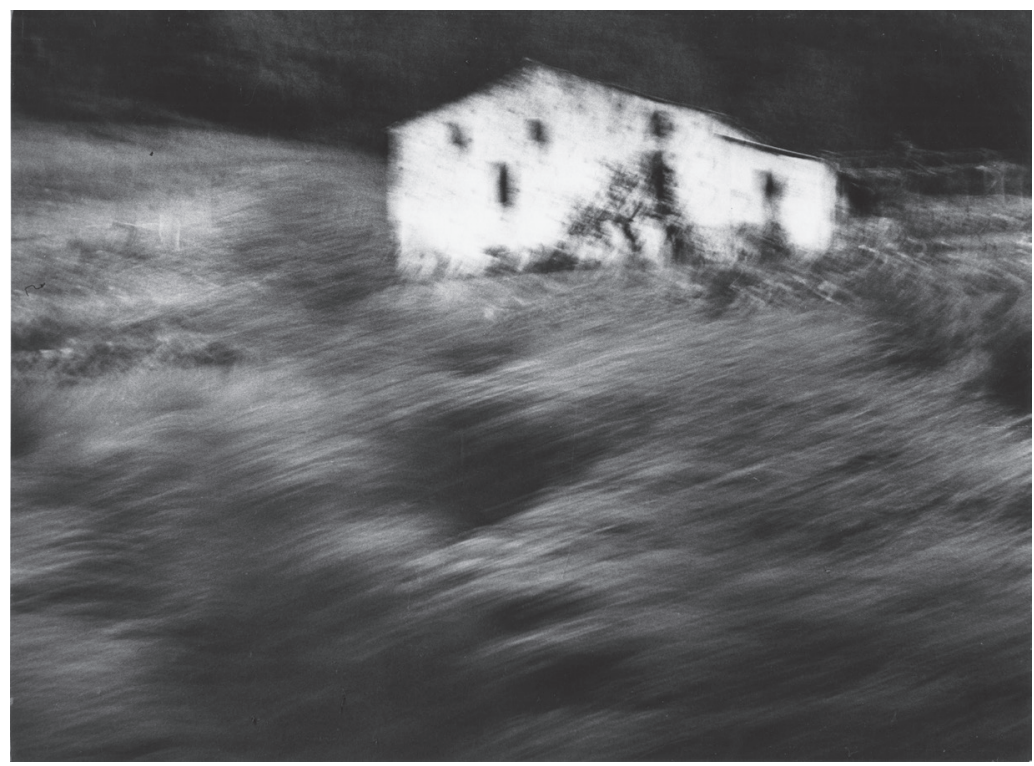

Fig. I: Mario Giacomelli, immagine 2 della serie La notte lava la mente, I994-1995.

Continuando la visione comparata, alle «fila d'anime» del terzo verso paiono alludere diversi tipi di presenze, in modo particolare delle sagome fantasmatiche ed evanescenti allineate sulla spiaggia o le successive figure in maschera schierate in fila. Rispetto alla terza strofa della poesia il ciclo offre altre corrispondenze, a partire dal comune ambiente marittimo; la presenza di un gatto colto in movimento è invece considerabile a tutti gli effetti quale «segno di vita», confermato ulteriormente dalle immagini che evocano il rapporto paterno di un adulto e un bambino sulla spiaggia, cui segue la ripresa aerea delle teste di alcuni bagnanti che punteggiano la superficie del mare. Un ulteriore richiamo alla poesia è costituito dalla ventiduesima stampa (fig. 2), caratterizzata dai toni soffusi della gamma tonale dei grigi, che esprime l'atmosfera di incanto e sospensione lirica mutuata dalla poesia mostrando una figura scura solitaria sulla spiaggia intenta a scrutare, con la mano portata alla fronte, il mare popolato di gabbiani. 


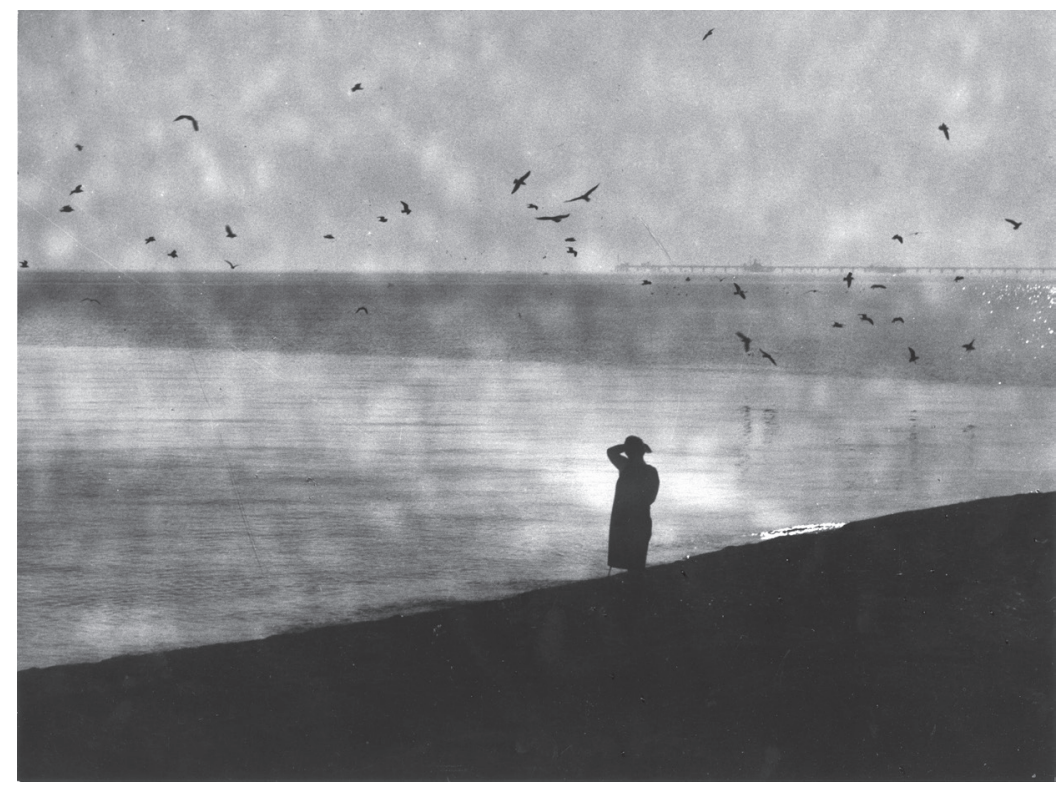

Fig. 2: Mario Giacomelli, immagine 22 della serie La notte lava la mente, I994-I995.

$\mathrm{Al}$ di là dei punti di contatto emersi da questo breve confronto, in linea generale entrambe le opere risultano simili a mosaici composti da singole immagini collegate fra loro da riferimenti allusivi: così come tra una strofa $\mathrm{e}$ l'altra della poesia, tra una coppia e l'altra di stampe la modalità di montaggio risponde spesso a criteri di tipo analogico, determinando una forma di spaesamento e di incertezza interpretativa.

Soffermandosi sui soggetti ritratti nelle immagini e ripercorrendone la storia all'interno della produzione giacomelliana emergono altri elementi che possono apportare ulteriori contributi esegetici. La casa bianca ritratta nel secondo scatto è infatti un soggetto su cui l'obiettivo di Giacomelli ha insistito per quarant'anni, come ricorda a proposito il figlio Simone: «[...] ogni domenica mattina in inverno, e il sabato pomeriggio in estate, fotografa sempre la stessa casa, in campagna, fino al 1995 anno in cui questa casa crolla. Mi portò da lei in visita, come da una persona cara, per salutarla e spiegarmi le ragioni del tempo» (2008: 197).

Secondo Alessandro Pellegrino, quella che l'artista ha sempre chiamato «la casa bianca», costituisce un «mito ricorrente che richiama le radici contadine dell'autore, luogo deputato dei sentimenti individuali e familiari che Giacomelli considera più sacri» (1997: 43). A questo proposito il fotografo ha infatti affermato: «[...] mi affascina questa casa che stiamo osservando poiché in questa luce che la natura dà, in questa materia io vedo allo stesso 
tempo la mia anima ma anche il contadino, vedo le sue speranze, quello che lui si aspetta e chiede alla terra» (Lucchini i994: I49). Il crollo, avvenuto in concomitanza con la composizione della serie luziana, diviene dunque l'emblema di un rapporto ferito tra l'uomo e la terra, il cui danneggiamento causato dall'agricoltura intensiva richiama a sua volta la disgregazione del tessuto di relazioni umane che era andato consolidandosi nei secoli.

Le fattezze antropomorfe della casa, avvolta da un'atmosfera sinistra, appaiono contratte in un grido di disperazione che denuncia la crisi di ciò di cui è simbolo, ossia di quei valori divenuti inattuali ai quali con la sua presenza ancora richiamava. L'effetto di mosso dell'immagine può essere ricondotto alla consuetudine di Giacomelli di fotografare nuovamente muovendo l'apparecchio le sue stesse stampe, a volte realizzate anni prima; una pratica che lo stesso artista ha motivato affermando che «muovere la macchina per me vuol dire far vivere un'immagine morta, cioè farla vivere oltre il tempo» (I990: 22).

Questa immagine introduce dunque nella serie la riflessione sulle «ragioni del tempo» e il suo travaglio, lo scorrere degli anni, il dolore e la paura generata dal corrompersi delle cose e dall'invecchiare delle persone. Come afferma Andreani, una delle tematiche peculiari di Giacomelli è rappresentata dalla vita intercettata soglia sul non-essere, come si vede sin dalle fotografie scattate all'ospizio o a Lourdes dove l'umanità è colta «a un soffio dalla propria scomparsa» (2019: I9).

Il tema della perdita, del crollo, si sviluppa poi nella serie in una coppia di immagini in cui sono ritratti uomini anziani ripresi attraverso un registro stilistico che infonde un senso di straniamento e inquietudine, mettendo in evidenza non la fisicità delle figure, che appare sbiadita, ma le loro ombre. A questi due scatti segue un'altra coppia in cui appaiono persone anziane assieme a soggetti vestiti con costumi tradizionali intenti a celebrare, con musiche e canti, la festa contadina di Cantamaggio: rievocazioni di un passato popolare in via di estinzione a cui Giacomelli guardava con un senso di rimpianto.

A questo punto viene introdotto il tema del travestimento e al motivo della riflessione nostalgica subentra una realtà grottesca contraddistinta dalla maschera e dalla finzione, a rappresentare la deriva dell'uomo contemporaneo ormai sradicato dalla propria terra e lontano dai suoi valori autentici. La prima delle due immagini vede i personaggi disposti in modo disordinato, mentre nella seconda le figure presenti sono in posizione statuaria, disposte a scacchiera, ammantate della loro solitudine e della loro esibita distanza. Quella messa a tema è perciò una realtà umana che si presenta smarrita e disgregata, come le lettere sparse per terra in uno dei primi scatti. 
Nell'immagine che segue, una maschera è disposta in primissimo piano e sembra interpellare con veemenza chi guarda, rivolgendo un'interrogazione violenta sulla falsità del vivere contemporaneo. Successivamente lo stesso artista si chiama in causa in prima persona autoritraendosi al fianco della maschera e mostrando sullo sfondo un edificio diroccato, probabilmente la stessa casa della seconda immagine. È questa la prima volta, dopo le primissime fotografie degli anni Cinquanta, che Giacomelli adotta la tecnica dell'autoritratto; qui l'autore sembra affermare che ognuno è chiamato a decidere tra la verità e la finzione, con alle spalle il crollo della casa, la consunzione dei valori.

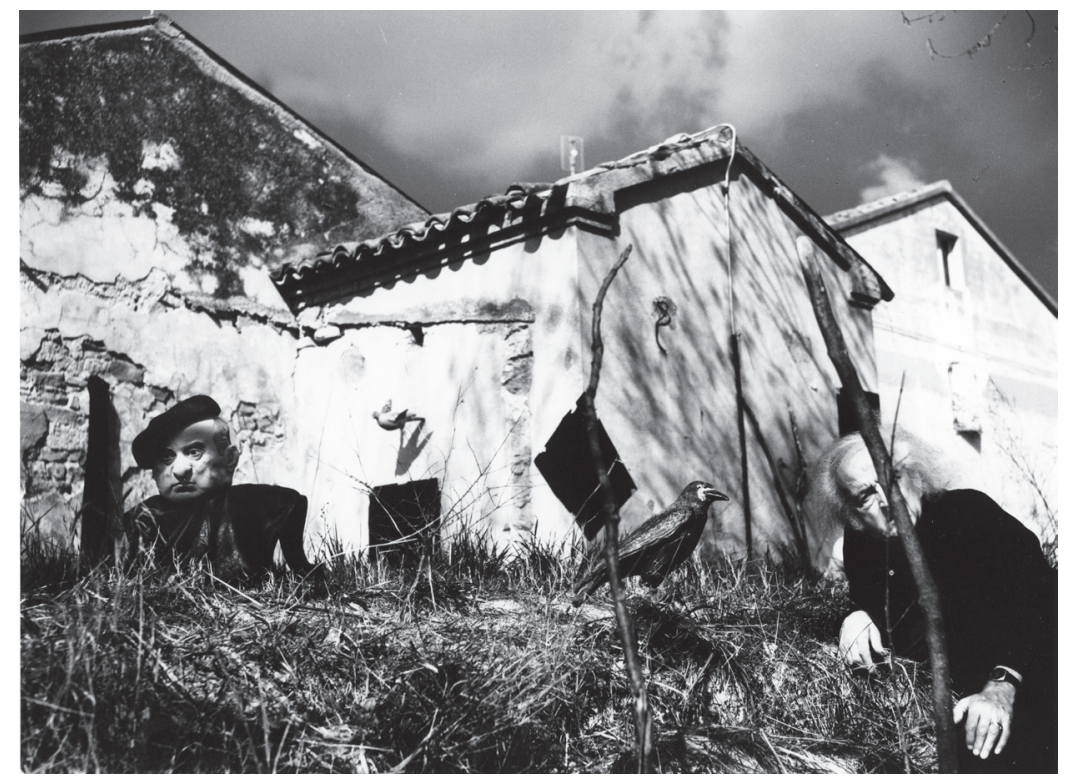

Fig. 3: Mario Giacomelli, immagine I2 della serie La notte lava la mente, I994-1995.

La tematica fin qui sviluppata da Giacomelli è comune ad altre serie dell'ultimo periodo, come si nota prendendo in considerazione il lavoro appena precedente, realizzato tra il 1992 e il 1994, dal titolo Io sono nessuno, tratto dall'omonima poesia di Emily Dickinson. L'interrogazione con cui comincia il testo «Io sono nessuno, tu chi sei?» anticipa la messa in discussione del proprio io osservata ne La notte lava la mente; nello stesso lavoro vengono inoltre introdotte pseudopresenze come animali finti (utilizzati anche nella serie luziana), spaventapasseri, ombre e sagome umane prive di una realtà corporea, o personaggi grotteschi di una tragicomica pantomima apparse precedentemente già nel 1986 nelle serie tratte dalle poesie di Permunian: su 
tutto domina l'assenza, la falsità, l'incomunicabilità e l'alienazione. Tornando a La notte lava la mente, nella seconda parte della serie il discorso sembra ammettere la possibilità di una rinascita o di un rinnovamento, rappresentati dall'immagine della barca di fronte al mare e dai primi passi di un bambino.

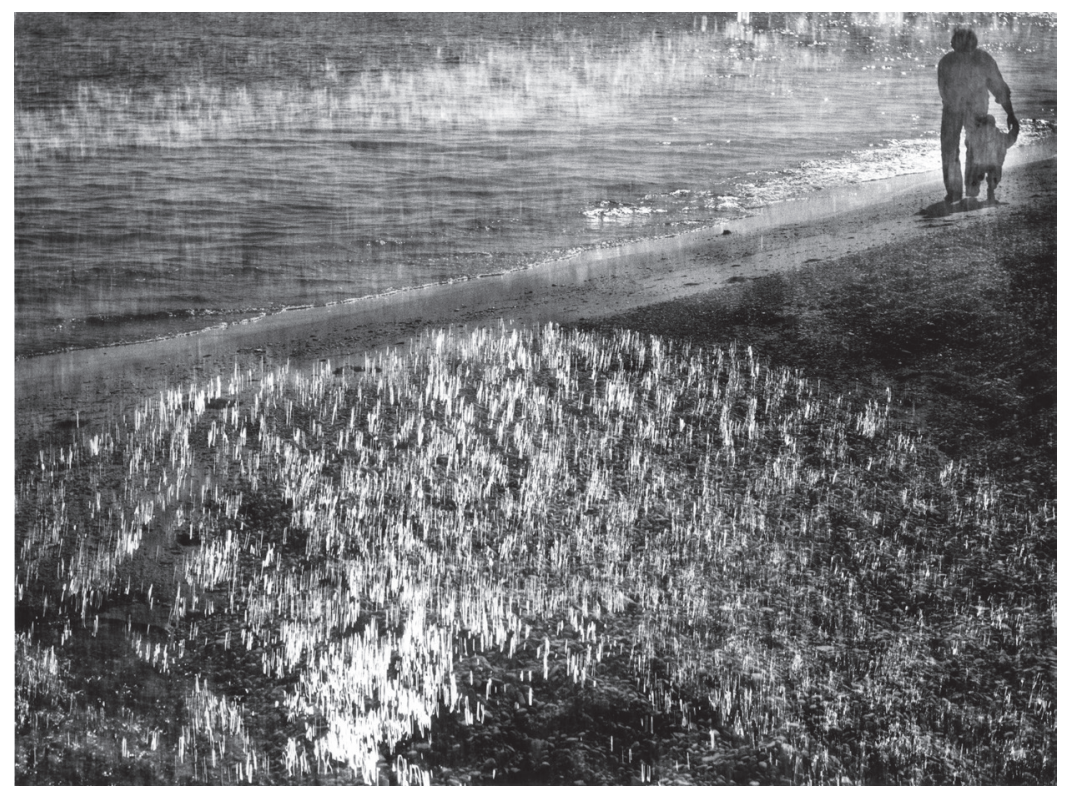

Fig. 4: Mario Giacomelli, immagine I5 della serie La notte lava la mente, I994-I995.

\section{Intrecci semantici}

Alla luce dell'analisi sin qui condotta, risulta evidente che i giudizi di Morello e Branzi riportati a inizio del lavoro, per cui gli approcci ai testi da parte di Giacomelli sarebbero frutto di una lettura superficiale o di una mera ricerca di legittimazione, siano quantomeno discutibili. Se infatti, come afferma lo stesso Morello (20I0: 394) facendo riferimento ad alcune lettere di Giacomelli, originariamente l'utilizzo del raffronto poetico può essere stato concepito come un tentativo di nobilitare la propria opera tramite l'assegnazione di un titolo dal sapore letterario o per supplire a una mancanza di tematiche nella realtà di Senigallia, è doveroso riconoscere che tra gli esiti riscontrabili nell'ultima parte della sua opera rientra il complesso intreccio di relazioni tra testo e immagini de La notte lava la mente.

Bisogna inoltre notare che, al di là delle dichiarazioni critiche in merito alle operazioni di Giacomelli, la realizzazione di fotografie associate a 
testi letterari si configura come una soluzione poco praticata dagli artisti e vista generalmente con perplessità da parte della critica. Afferma ad esempio John Berger che «quel che così spesso ostacola qualsiasi flusso, quando immagini e testo sono usati insieme, è la tautologia, la tediosa ripetizione di una stessa cosa detta due volte, la prima a parole e la seconda attraverso un'immagine» (20I4: 204). Il motivo di impasse è stato delineato anche da Arturo Carlo Quintavalle riferendosi al gruppo di fotografie che Ugo Mulas ha realizzato a partire dal 1962 ispirandosi agli Ossi di seppia montaliani. Afferma infatti il critico:

Il problema era [...] come illustrare un tema affatto letterario, anzi il problema reale era se, o meno, illustrare, traducendo le immagini di Montale in figure. Sarebbe stato, agire così, una mera prosecuzione del modello consueto di volume illustrato dove l'icone è una specie di commento pleonastico al testo, l'equivalente simmetrico della didascalia apposta a una qualsiasi immagine (I983: 219).

Bisognerebbe dunque chiarire se un'opera fotografica possa riferirsi a un testo letterario senza ridursi a illustrazione, ovvero a una sorta di ekphrasis rovesciata a esso del tutto subalterna.

La stessa obiezione posta in merito alla funzione illustrativa della fotografia si pone anche in relazione alle conseguenze di tale rapporto nei confronti delle possibilità semantiche della parola poetica. Secondo Clement Greenberg infatti, rispetto ai rapporti tra poesia e immagine (qui generica, non specificamente fotografica), se una delle possibilità di significato destate dalla finzione letteraria «dovesse essere realizzata con troppa precisione, la poesia perderebbe la maggior parte della sua efficacia, che consiste nello smuovere la coscienza con infinite possibilità, accostandosi all'orlo del significato senza mai giungervi pienamente» (I940: 6I). Le infinite realizzazioni possibili della parola si potrebbero dunque ridurre a un unico e definito esito visivo, pietrificato dall'occhio fotografico della medusa ${ }^{8}$.

Per affrontare questo aspetto occorre ammettere che la potenzialità semantica del testo poetico, l'irradiarsi dei suoi possibili significati suscita naturalmente l'immaginario del lettore, come sostiene Andrea Cortellessa:

[...] la parola poetica in quanto tale - rispetto alla compattezza argomentativa della prosa - mantiene sempre un che di mutilo, di incompiuto. Per sua natura essa si rivolge a chi legge in cerca di un completamento di senso da parte sua, qualcosa che è dunque a sua volta un adempimento (20IO: 4IO).

8 L'immagine è presa in prestito dal titolo del volume di Remo Ceserani (20II). 
Se, come nota anche Piero Bigongiari, di per sé la «parola chiede al lettore di specificarsi visivamente» (I999: II6), allo stesso modo, Michele Cometa evidenzia che «ogni immagine fotografica in fin dei conti reclama un testo» (20I6: 72). In questa dimensione di evocazione reciproca, appare tutt'altro che singolare l'intenzione artistica di produrre una realizzazione visiva di una poesia, anche per mezzo di una ripresa fotografica. Stando a quanto afferma George Steiner introducendo il concetto di «mislettura» (I998: I23), la lettura di un testo potrà essere limitata o addirittura abusiva, ma sarà comunque un apporto fertile se darà vita a una nuova opera in relazione al testo di partenza.

Per il critico francese, questo tipo di mislettura operativa è l'unica azione ermeneutica feconda che la poesia richiede e si configura come autentico atto critico (I998: 24), confrontandosi con ciò che lo ha preceduto e divenendo a sua volta potenzialmente generativo. Siamo davanti a un tentativo di interpretazione e riformulazione che rientra in ciò che Massimo Carboni definisce un'«ermeneutica operativa [...] una pratica eminentemente dialogica in cui l'artista dimostra da parte sua (e con i suoi mezzi specifici) che la storia alle sue spalle può tornare ed essere produttiva se ricompresa e riesposta in un gesto» (2002: 132-133).

In tal senso, il caso affrontato dal presente studio è emblematico: non solo Giacomelli ha composto la propria serie in dialogo con la poesia, ma lo stesso Luzi ha scritto La notte lava la mente a partire dal confronto con la seconda cantica dantesca, dando dunque vita a un palinsesto testuale di tre livelli. La dinamica della riscrittura genera una tessitura di rimandi, come afferma Gérard Genette sostenendo che «l'arte di "fare il nuovo col vecchio" ha il vantaggio di creare oggetti più complessi dei prodotti "fatti apposta": una funzione nuova si sovrappone e si intreccia a una struttura vecchia, e la dissonanza fra questi due elementi in compresenza rende stimolante l'insieme» (1997: 468-469).

Rimangono però a questo punto ancora irrisolte le obiezioni di Berger, Quintavalle e Greenberg riportate a inizio del paragrafo; per affrontare l' impasse illustrativa è utile interrogare la serie di Giacomelli presa in esame. $\mathrm{Ne}$ La notte lava la mente la problematica legata al rapporto tautologico tra i due media sembra essere scongiurata. Come già riportato dalle parole dell'autore nella prima parte del lavoro, Giacomelli non intendeva operare una trasposizione della poesia per immagini, ma esprimere tramite un racconto fotografico sentimenti suscitati dai versi; la posizione dell'autore è dunque libera dal concetto di fedeltà al testo di partenza e non è da interpretarsi quale una traduzione in immagini dei versi.

Un altro elemento da considerare è rappresentato dalla relazione tra l'enigmaticità della serie e l'indefinitezza delle immagini, che non si caratterizzano per chiarezza denotativa, bensì per un effetto straniante e a vol- 
te ambiguo. Di fronte all'alterazione della realtà ritratta di una fotografia anti-mimetica, autentica cifra stilistica di Giacomelli, la qualità referenziale delle stampe passa in secondo piano ${ }^{9}$ rispetto all'atmosfera suggerita o all'investitura simbolica dei soggetti, oltre a essere in rapporto alla sua collocazione nello svolgimento della narrazione, come si può notare ad esempio negli scatti che mostrano la pioggia di luce.

Si rileva poi che la poesia non è connessa a un'unica immagine, bensì a un racconto fotografico, per cui ogni ipotesi ermeneutica nasce dalla negoziazione dei significati tra le immagini, connesse l'una all'altra attraverso risonanze contenutistiche e formali; all'interno del montaggio ogni scatto, dunque, diviene una cellula vitale di un'articolazione di segni capace di generare una proliferazione di significati. Le relazioni con i versi possono inoltre vivere di volta in volta momenti di maggiore o minore vicinanza, oscillando da un rapporto illustrativo all'introduzione di elementi non riscontrabili nei versi, in uno sviluppo narrativo che rende dinamico il rapporto intermediale delineando nuovi orizzonti semantici.

Il testo poetico e quello visivo non si fondono, ma restano connessi in una dimensione fototestuale dove l'esperienza artistica completa delle immagini si genera dal confronto con i versi di Luzi. Secondo categorie approntate da Umberto Eco (20I0: 373-375), ci troviamo di fronte a un fenomeno di adattamento o trasmutazione per manipolazione di un ipotesto verbale (mantenuto nella dimensione paratestuale del titolo) il cui esito è un ipertesto visuale. In questo accostamento si crea un reciproco scambio produttivo, giacché le potenzialità della poesia vengono amplificate alla luce delle possibilità interpretative irradiate dalle immagini; l'attenzione dell'osservatore oscilla quindi dal testo di partenza a quello di arrivo per poi tornare a quello di partenza, arricchito del contributo di adempimenti possibili della parola poetica. Un contributo plurimo e polisemantico, non così specificamente determinato da imporsi come via univoca e precludere ulteriori esiti alternativi.

Giacomelli, attraverso quello che Linda Hutcheon potrebbe ricondurre a un orizzonte di adattamento, mette in atto un «ampio confronto intertestuale con l'opera» mediante «sia una (re) interpretazione che una (ri) creazione» (20II: 28). Il fotografo sembra aver usato il testo come catalizzatore immaginifico per cui, a partire da agganci testuali e sorretto da una griglia formale sotterranea mutuata dalla poesia, ha raccolto l'elemento temporale del transito per sviluppare una riflessione sul trascorrere del tempo in linea con la ricerca dell'ultima parte della sua produzione, senza seguire fedelmente la traiettoria semantica dei versi.

9 Sandro Genovali in merito alla fotografia di Giacomelli afferma che «questo potere di intervenire sulla realtà è così drastico e dittatoriale da mettere persino in discussione la natura stessa della fotografia, quello che si dice il suo "specifico"» (I978). 
Allontanandosi dunque dalla lirica, egli ha messo in luce piuttosto l'angoscia di fronte al venir meno di una vita autentica, sostituita da una condotta falsa; una possibile interpretazione giacomelliana del verso «chi pronto al balzo, chi quasi in catene» può infatti riferirsi alla scelta tra un modo di vivere autentico ma inattuale e l'opzione alienante della finzione. In altre parole Giacomelli ha costruito la propria serie tenendo sottotraccia la lirica, nutrendola poi di elementi che caratterizzano il suo immaginario e dando voce alle proprie preoccupazioni.

Il risultato è un sistema aperto di dialogo tra testo e immagini all'interno di una sfera semiotica complessa, dove il testo di partenza nella ricezione e riformulazione attraverso un linguaggio non verbale viene totalmente reinventato. La visione di Giacomelli mette dunque in atto un adempimento della parola poetica che dà origine a una mutua integrazione e a un potenziamento reciproco di significato, aprendo la strada a ulteriori, possibili narrazioni.

\section{Appendice}

Si elencano di seguito, in ordine cronologico, le serie fotografiche di Giacomelli il cui titolo è stato offerto da un testo poetico:

Verrà la morte e avrà i tuoi occhi, I954-I968 (fino al I965 col titolo Vita d'ospizio); da Cesare Pavese.

Io non ho mani che mi accarezzino il volto, I96I-I963 (conosciuta anche come Pretini); da David Maria Turoldo.

A Silvia, I964 e I988; da Giacomo Leopardi.

Caroline Branson da Spoon River, I967 e I97I-I973; da Edgar Lee Masters.

Il canto dei nuovi emigranti, I984; da Franco Costabile.

Il teatro della neve (che comprende Luna vedova per le strade di mare e Ho la testa piena, mamma) I984-I986; da Francesco Permunian.

Ninna nanna, I985-1987; da Léonie Adams.

Felicità raggiunta, si cammina, I986-I992; da Eugenio Montale.

L'infinito, I986-I988; da Giacomo Leopardi.

Passato, I986-I990; da Vincenzo Cardarelli.

Io sono nessuno!, I992-I994; da Emily Dickinson.

La notte lava la mente, I994-I995; da Mario Luzi.

Diversi? Ti chiesi, 1997 (serie inedita); da Simone Giacomelli.

Bando, I997-I999; da Sergio Corazzini.

La mia vita intera, I998-2000; da Jorge Luis Borges.

Ritorno, I999-2000 (mai esposta, edita in piccolo formato in Giacomelli 2009: 246-247); da Giorgio Caproni. 


\section{Bibliografia e sitografia}

Amodeo, Fabio - Giusa, Antonio - Turrin, Raffaella (a cura di), Luigi Crocenzi. Un racconto per immagini, Spilimbergo, CRAF, 2013.

Andreani, Marco, «Alle fonti della poesia: le liriche leopardiane nelle fotografie di Mario Giacomelli», in Giacomelli 20I9, pp. I6-26.

Bigongiari, Piero, «Ut poesis pictura» [1982], in Id., La poesia pensa. Poesie e pensieri inediti. Leopardi e la lezione del testo, a cura di E. Biagini - P.F. Iacuzzi - A. Noferi, Firenze, L.S. Olschki, I999, pp. II6-I30.

Biondi, Katiuscia, «Sotto la pelle del reale», in Ead. - M. Itolli - C. Zucchetti (a cura di), Mario Giacomelli. Sotto la pelle del reale, con prefazione di A. Bonito Oliva, Milano, 24 Ore Cultura, 20II, pp. I3-2I.

Carboni, Massimo, L'occhio e la pagina. Tra immagine e parola, Milano, Jaca Book, 2002.

Ceserani, Remo, L'occhio della medusa. Scrittori e fotografi, Torino, Bollati Boringhieri, 201 .

Cometa, Michele, «Forme e retoriche del fototesto letterario», in Id. - R. Coglitore (a cura di), Fototesti. Letteratura e cultura visuale, Macerata, Quodlibet, 2016.

Cortellessa, Andrea, «L'ombra, l'opera», in C. Parmiggiani, Una fede in niente ma totale, a cura di A. Cortellessa, prefazione di J.-L. Nancy, Firenze, Le lettere, 20I0, pp. 397-4I5.

Eco, Umberto, Dire quasi la stessa cosa. Esperienze di traduzione [2003], Milano, Bompiani, 2010.

Genette, Gérard, Palinsesti. La letteratura al secondo grado [I982], Torino, Einaudi, 1997.

Genovali, Sandro, «Un artista senigalliese, Mario Giacomelli», Controvento, agosto I978; ora in A.C. Quintavalle (a cura di), Mario Giacomelli, Milano, Feltrinelli, I980, p. 245.

Giacomelli, Mario, Spazi interiori, a cura di E. Carli, Ancona, Adriatica Editrice, I990.

-. La notte lava la mente, Milano, Photology, 1997.

-. Mario Giacomelli, a cura di G. Celant, catalogo della retrospettiva al Palazzo delle Esposizioni, Roma, Photology-Logos, $200 \mathrm{.}$

- La mia vita intera, a cura di S. Guerra, prefazione di C. Colombo, Milano, Mondadori, 2008.

-. La figura nera aspetta il bianco, a cura di A. Mauro, Roma, Contrasto, 2009.

-. Mario Giacomelli. Giacomo Leopardi, L'Infinito, A Silvia, a cura di A. Giampaoli - M. Andreani, Cinisello Balsamo, Silvana, 2019.

Giacomelli, Simone, «Mario Giacomelli. Ovvero i ricordi di un ragazzo nato nel I925 e di suo figlio nato nel I968», in Giacomelli 2009, pp. 7-39.

Goethe, Johann Wolfgang, Massime e riflessioni, Milano, Rizzoli, 2013. 
Greenberg, Clement, «Verso un più nuovo Laocoonte» [I940], in Id., L'avventura del modernismo. Antologia critica, a cura di G. Di Salvatore e L. Fassi, Milano, Johan \& Levi, 20II, pp. 52-64.

Guerra, Simona, Parlami di lui. Le voci di Scianna, Berengo Gardin, Ferroni, Camisa, Colombo, Branzi, Manfroi, De Biasi, Permunian, Biagetti, su Mario Giacomelli, Ancona, Mediateca delle Marche, 2007.

Hutcheon, Linda, Teoria degli adattamenti. I percorsi delle storie tra letteratura, cinema, nuovi media [2006], Roma, Armando, 201.

Luzi, Mario, Lopera poetica, a cura e con un saggio introduttivo di S. Verdino, Milano, Mondadori, I998.

—. «Purgatorio. La notte lava la mente» [1990], in Id., Teatro, Milano, Garzanti, pp. 4I5-494.

M.[ascellani], E.[lisabetta], «Mario Giacomelli. Il poeta delle immagini», Percorsi in chiaroscuro, II, I, Io febbraio I997, pp. 37-40; ora in Giacomelli 200I, p. 84.

Morello, Paolo, La fotografia in Italia 1945-I975, Roma, Contrasto, 20 IO.

Pellegrino, Alessandro, «La casa dell'essere. Poesia e fotografia in Mario Giacomelli», Rocca. Rivista della Pro Civitate Christiana, 4, I5 febbraio I997, pp. 42-43.

Quintavalle, Arturo Carlo, Messa a fuoco. Studi sulla fotografia, Milano, Feltrinelli, I983.

Ria, Antonio, «Fotografia come poesia. Incontro con Mario Giacomelli» [1997], in Id. (a cura di), Mario Giacomelli. Enzo Cucchi. Nati in un fosso, Lugano, Le ricerche, 2003, pp. 38-46.

Steiner, George, Vere presenze. Contro la cultura del commento, una difesa dell'arte e della creazione poetica [I989], Milano, Garzanti, 1998.

Archivio Mario Giacomelli (C Rita Giacomelli: https://www.archiviomariogiacomelli.it

Per tutte le immagini Courtesy Archivio Mario Giacomelli (C Rita Giacomelli. 
